\title{
«Nach dem Abstimmungserfolg kommt die Knochenarbeit»
}

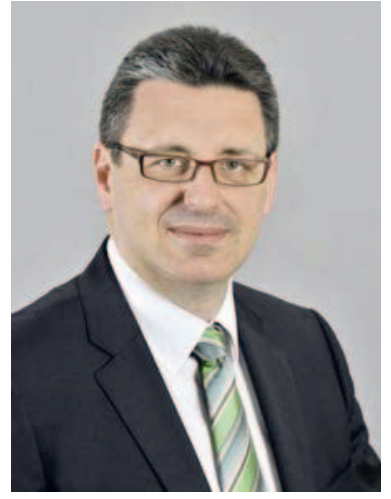

Martin Bangerter,

designierter Co-Präsident

Dachverband Komplementärmedizin Präsident Schweizerischer Drogistenverband $(S D V)$

\section{Martin Bangerter}

Der Dachverband Komplementärmedizin (Dakomed) blickt auch 2012 auf ein konstruktives Jahr zurück. Die verlässliche Interessenvertretung gegenüber der Politik bringt Fortschritte in der Berücksichtigung und Anerkennung der Komplementärmedizin im schweizerischen Gesundheitswesen. Wie in der Politik üblich, gibt es in mehreren Dossiers zeitliche Verzögerungen. Ein Grund hierfür sind unter anderem die Wechsel im Eidgenössischen Departement des Inneren (EDI) (neuer Gesundheitsminister, neue persönliche Mitarbeitende und Dossierverantwortliche) sowie im Bundesamt für Gesundheit (BAG). Erfreulich ist, dass der neue Gesundheitsminister Alain Berset der Komplementärmedizin gegenüber sehr aufgeschlossen ist. Er hatte sich im Abstimmungskampf im Freiburger Kantonal-Komitee engagiert.

Der Dachverband setzt sich für die Berücksichtigung der Komplementär- und Alternativmedizin im schweizerischen Gesundheitswesen ein. Sein Ziel ist insbesondere die Umsetzung der mit Artikel 118a «Komplementärmedizin» der Bundesverfassung verbundenen Kernforderungen:

- Förderung der integrativen Medizin (Zusammenarbeit von Schul- und Komplementärmedizin)

- Aufnahme ärztlicher Richtungen der Komplementärmedizin in die Grundversicherung und in die weiteren Sozialversicherungen (SUVA, Militär- und Invalidenversicherung)

- Förderung von Lehre und Forschung
- Schaffung nationaler Diplome und kantonaler Berufszulassungen für nichtärztliche Therapeuten

- Sicherstellung der Heilmittelvielfalt

\section{Leistungsausweis}

Seit dem 1. Januar 2012 werden die ärztlichen Methoden der Komplementärmedizin befristet bis 2017 wieder von der Grundversicherung vergütet. Für die definitive Aufnahme in den Leistungskatalog der Obligatorischen Krankenpflegeversicherung (OKP) sind Ergänzungen der Anträge der Fachgesellschaften sowie vonseiten des BAG ein Health Technology Assessment (HTA) geplant.

\section{Treffen mit Bundesrat Alain Berset}

Am 5. Juni 2012 hat eine Delegation des Dakomed dem Gesundheitsminister einen Antrittsbesuch abgestattet. Das Gespräch hat in einer guten Atmosphäre stattgefunden. Bundesrat Berset scheint gewillt zu sein, die Kernforderungen umzusetzen.

Die Ausgangslage habe sich nach der Volksabstimmung verändert, sagte der Bundesrat. Er ist bereit, Verbindlichkeit bezüglich der Spielregeln zur Aufnahme einer Leistung in die Grundversicherung zu schaffen.

Bei den Heilmitteln bekannte der Bundesrat, dass die Thematik komplex sei und er sie noch wenig kenne. Ständerat Luc Recordon wies auf die Bedeutung der nichtärztlichen Therapeuten im Gesundheitswesen und die laufenden Diplomierungsarbeiten beim Bundesamt für Berufsbildung und Technologie (BBT) hin.

Semya Ajoubi (Leiterin Begleitgruppe) verlässt das EDI in Richtung Eidgenössisches Departement für auswärtige Angelegenheiten (EDA). Bundesrat Berset bestätigte, dass die Arbeiten der Begleitgruppe weitergehen und dass er für heikle Fragen zur Verfügung steht.

\section{Begleitgruppe Komplementärmedizin}

Die Begleitgruppe Komplementärmedizin des EDI wird neu von Frau Katharina Füglister geleitet. Leider fand unter der neuen Leitung noch kein Treffen statt. Frau Füglister hat aber versichert, Anfang des Jahres 2013 endlich die lang ersehnte und dringend notwendige Sitzung einzuberufen. Die Union komplementärmedizinischer Ärzteorganisationen wartet noch immer auf eine verbindliche Rückmeldung zu ihrem im Sommer 2012 eingereichten Evaluationskonzept zur Überprüfung der Anträge für die definitive Aufnahme in der Grundversicherung.

\section{KARGER}




\section{Ständerat Joachim Eder neuer Co-Präsident}

\section{der parlamentarischen Gruppe Komplementärmedizin}

Ständerat Joachim Eder FDP ZG ersetzt den zurückgetretenen bisherigen Co-Präsidenten alt Ständerat Rolf Büttiker FDP SO. Gemeinsam mit Nationalrätin Edith Graf-Litscher SP TG leitet er die parlamentarische Gruppe Komplementärmedizin. Joachim Eder war vor seiner Wahl in den Ständerat Gesundheitsdirektor im Kanton Zug. Mit ihm gewinnen wir einen ausgewiesenen Gesundheitspolitiker für die parlamentarische Gruppe. Die parlamentarische Gruppe setzt sich aus 15 Ständerätinnen und Ständeräten (früher 8) und 45 Nationalrätinnen und Nationalräten (früher 37) aller Parteien zusammen.

\section{Heilmittelgesetz und Vorstösse zur Sicherung} der Arzneimittelvielfalt eingereicht

Der Bundesrat hat am 14. November 2012 die Botschaft und den Revisionsentwurf des Heilmittelgesetzes (2. ordentliche Revisionsetappe) verabschiedet. Wichtige Anliegen des Dakomed wurden darin berücksichtigt. So beispielsweise die Kleinmengenlösung (100 Packungen, 3000 Tagesdosen), die vereinfachte Zulassung für nicht verschreibungspflichtige Arzneimittel mit Indikationsangabe (30/15 Jahre) und die Zulassung aufgrund einer Meldung (Komplementärarzneimittel ohne Indikationsangabe, deren Wirkstoffe in Listen zu speziellen Therapierichtungen aufgeführt sind). Aus unserer Sicht zu erweitern ist die Definition von Phytoarzneimitteln. Es braucht eine Kongruenz zur EU-Gesetzgebung, wo Kombinationen mit Mineralien und Vitaminen zulässig sind.

In der Herbstsession 2012 haben Mitglieder der parlamentarischen Gruppe Komplementärmedizin im Parlament Vorstösse zum Erhalt der Arzneimittelvielfalt in der Schweiz eingereicht. Die Zahl der zugelassenen Arzneimittel nimmt seit Jahren ab, weil die Zulassungshürden hoch sind und Innovationen verhindert werden. Folgende Vorstösse wurden deponiert:

- 12 3788: Postulat Joachim Eder. Arzneimittel, Nahrungsergänzungsmittel und Medizinprodukte. Ungenügende Vorgaben, Friktionen im Vollzug.

- 12 3789: Motion Joachim Eder. Bürokratieabbau bei genehmigungspflichtigen und meldepflichtigen Änderungen von Arzneimitteln.

- 12 3822: Postulat Edith Graf-Litscher. Zu strenge Zulassungshürden für Arzneimittel der Komplementärmedizin.
- 12 3824: Postulat Edith Graf-Litscher. Massnahmen gegen die Abnahme von zugelassenen pflanzlichen und komplementärmedizinischen Heilmitteln.

- 12 3847: Motion Yvonne Gilli. Arzneimittelvielfalt in der Komplementärmedizin erhalten.

- 12 471: Parlamentarische Initiative Yvonne Gilli. Erneute Verlängerung der kantonalen Zulassung von Arzneimitteln.

\section{Medizinalberufegesetz}

Die Botschaft zum vernehmlassten Medizinalberufegesetz (MedBG) wurde auf den Sommer 2013 verschoben. Erfreulich ist, dass die Mehrheit der Vernehmlassungsteilnehmer die Integration von Komplementärmedizin in das Studium der Medizin und Pharmazie befürwortet.

\section{Nationale Diplome für KomplementärtherapeutInnen und} NaturheilpraktikerInnen in Reichweite

Die beiden Organisationen der Arbeitswelt Alternativmedizin (OdA AM) und KomplementärTherapie (OdA KT) haben 2012 ihre jeweiligen Berufsbilder, die eine Beschreibung ihrer Arbeitsfelder, beruflichen Grundlagen und Kompetenzen enthalten, ausgearbeitet und verabschiedet. Aktuell arbeiten beide Organisationen an den Prüfungsordnungen, und Pilotprüfungen sind für den Herbst 2013 vorgesehen.

\section{Workshop Grundversorgung - ein Grund zur Sorge?}

Der Dakomed hat im Juni 2012 an einem Workshop darüber diskutiert, wie eine sichere und qualitativ hochstehende Grundversorgung aussehen könnte. Die Versorgungsmodelle der Zukunft stellen den Patienten als Ganzes ins Zentrum, sind interdisziplinär und vernetzt. Die Gesundheitsakteure dürfen nicht auf die Politik warten, sondern müssen selber aktiv werden. Dies das Fazit einer Veranstaltung.

\section{Komplementärmedizin - ein Leitfaden für Patientinnen und Patienten}

Zur Stärkung der Patientenkompetenz im Bereich der Komplementärmedizin hat der Dakomed einen Leitfaden entwickelt. Dieser soll Patientinnen und Patienten eine Entscheidungshilfe für die Anwendung von Komplementärmedizin sein. Er möchte allen Patienten Mut machen, mit ihrer Ärztin, ihrer Naturheilpraktikerin bzw. ihrer Komplementärtherapeutin, ihrer Drogistin oder Apothekerin über ihre Wünsche und Anliegen zu sprechen. 


\section{Herausforderungen 2013}

\section{Heilmittelgesetzrevision}

Die Beratung des revidierten Heilmittelgesetzes beginnt voraussichtlich im Frühjahr 2013. Der Dakomed wird sich dafür einsetzen, dass dieses den Anliegen der Komplementärmedizin Rechnung trägt.

\section{Medizinalberufegesetz}

Die Botschaft zum vernehmlassten MedBG wurde auf den Sommer 2013 verschoben. Erfreulich ist, dass die Mehrheit der Vernehmlassungsteilnehmer die Integration von Komplementärmedizin in das Studium der Medizin und Pharmazie befürwortet.

\section{Lehre und Forschung}

Die Komplementärmedizin ist an den Schweizer Universitäten noch immer untervertreten. Der Dakomed hat für
2013 die Lancierung einer kantonalen Volksinitiative zur Schaffung eines Lehrstuhls für Komplementärmedizin an der Universität Basel beschlossen.

\section{Internationale Vernetzung}

Der Dachverband beobachtet die internationalen Entwicklungen in der Komplementärmedizin und pflegt vermehrt Kontakte zu Partnerorganisationen in Europa.

\section{Fazit}

Die vollständige Umsetzung des Verfassungsartikels und des Volkswillens wird seine Zeit brauchen. Der Dachverband arbeitet weiterhin mit Nachdruck daran, dass Bund und Kantone die Komplementärmedizin im Gesundheitswesen angemessen berücksichtigen. 